\title{
Solution-processable, photo-stable, low-threshold, and broadly tunable thin film organic lasers based on novel high-performing laser dyes
}

\author{
María A. Díaz-García*a, Marta Morales-Vidala ${ }^{\mathrm{a}}$, Manuel G. Ramírez ${ }^{\mathrm{a}}$, José M. Villalvilla \\ Pedro G. Boj ${ }^{\mathrm{b}}$, José A. Quintana ${ }^{\mathrm{b}}$, A. Retolaza ${ }^{\mathrm{c}}, \mathrm{S}$. Merino $^{\mathrm{c}}$ \\ ${ }^{a}$ Dept. Física Aplicada and Instituto Universitario de Materiales de Alicante (IUMA), Universidad de

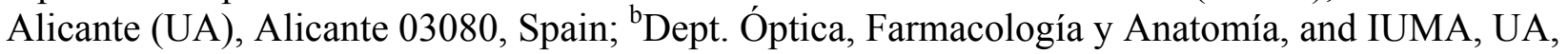 \\ Alicante 03080, Spain; \\ ${ }^{c}$ Micro and Nano Fabrication Unit, IK4-Tekniker, Eibar 20600, Spain
}

\begin{abstract}
Thin film organic lasers (TFOLs) represent a new generation of inexpensive, mechanically flexible devices with demonstrated applicability in numerous applications in the fields of spectroscopy, optical communications and sensing requiring an organic, efficient, stable, wavelength-tunable and solution-processable laser material. A distributed feedback (DFB) laser is a particularly attractive TFOL because it shows single mode emission, low pump energy, easy integration with other devices, mechanical flexibility and potentially low production cost. Here, amplified spontaneous emission (ASE) and DFB laser applications of novel high performing perylene dyes and $p$-phenylenevinylene (PV) oligomers, both dispersed in thermoplastic polymers, used as passive matrixes, are reported. Second-order DFB lasers based on these materials show single mode emission, wavelength tunability across the visible spectrum, operational lifetimes of $>10^{5}$ pump pulses, larger than previously reported PV oligomers or polymers, and thresholds close to pumping requirements with light-emitting diodes.
\end{abstract}

Keywords: organics, lasers, thin film, laser dyes, oligo( $p$-phenylenevinylene)s, perylenes, nanoimprint lithography, distributed feedback.

\section{INTRODUCTION}

Optically-pumped organic lasers have received great attention for various decades, being the capability of tuning the emission wavelength within the visible range their most interesting property with respect to other types of lasers. Actually, optically-pumped liquid dye lasers, with the active material consisting of a liquid solution containing a laser dye, are commercially available and often used in Spectroscopy, Photochemistry, Photophysics, nondestructive testing and Medicine. Due to important limitations of these devices, i.e. large size, need of high power pumping sources, difficulties in handling due to the need of recirculating the liquid solution to prevent dye photodegradation, etc., researchers have pursued for years the development of compact and easy-to-handle organic solid-state lasers (OSLs). ${ }^{1,2}$ Specially important towards achieving compactness, mechanical flexibility and easy integration with other devices, are thin film OSLs (TFOLs), among which, those that can prepared by means of solution-based methods, such as spincoating, printing, etc., are preferred for their prospect of reducing device cost. $^{2}$

The distributed feedback (DFB) laser, ${ }^{1,2}$ consisting of a waveguide film which includes a relief grating, has been a particularly successful TFOL in various applications in the fields of spectroscopy, ${ }^{3,4}$ optical communications ${ }^{2}$ and sensing. ${ }^{5,6}$ It presents several advantages: it provides single mode emission, which is important for certain applications; it requires low pump energy for operation, thus enabling pumping with compact and cheap sources such as laser diodes or even light emitting diodes (LEDs); the resonator can be easily integrated into other devices; it can be mechanically flexible; has a potentially low production cost; and can be easily integrated with field-effect-transistor geometry, promising for the development of electrically-pumped TFOLs. Grating fabrication is generally accomplished by techniques such as electron beam lithography (EBL), holographic lithography (HL), nanograting transfer or nanoimprint lithography (NIL), ${ }^{2}$ the latter showing the highest potential for fabrication scalable to mass production. In Figure 1 different possible device configurations with resonators fabricated by different methods are shown.

One of the major challenges remaining for the realization of a commercial compact and inexpensive TFOL is the active organic material, which should be simultaneously: i) efficient for lasing at a low threshold in order to operate under

Organic Light Emitting Materials and Devices XIX, edited by Franky So, Proc. of SPIE Vol. 9566, 95660Q (C) 2015 SPIE · CCC code: $0277-786 X / 15 / \$ 18 \cdot$ doi: 10.1117/12.2187015 
excitation with a weak light source; ii) photostable to ensure a long operational lifetime for the device; iii) capable of emission at various wavelengths or colors (color tuning); (iv) solution-processable, and (v) for the case of materials in which the organic units are dispersed in a matrix, show high miscibility into it. In addition, if the TFOL involves light propagation along the film plane, as in a DFB laser, the film should be properly prepared as to constitute a waveguide. The easiest way to assess the potential of a given material for a waveguide-based laser device is to study its amplified spontaneous emission properties (ASE). ${ }^{1,2}$ For a good ASE performance, a low-loss waveguide film with high photoluminescence (PL) efficiency is required, being both aspects highly dependent on the type of laser dye, host matrix and substrate material, as well as on parameters such as the dye doping ratio, the film thickness and the refractive index difference between film and substrate. Therefore, proper material selection and parameter adjustments are crucial to optimize the active medium before its inclusion in the laser cavity.

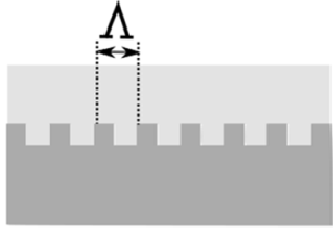

(a)

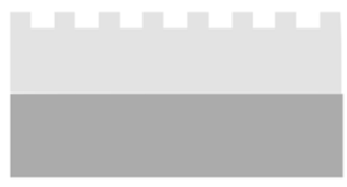

(b)

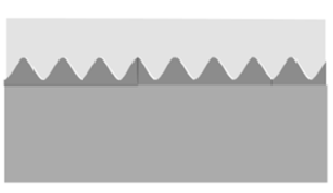

(c)

Figure 1. Different types of DFB laser geometries: (a) Grating on inorganic substrate (e.g. $\mathrm{SiO}_{2}$, fused silica or glass) fabricated by EBL, NIL or HL and subsequent etching; active film on top of it; (b) Grating on active film fabricated by NIL; (c) Grating on resist layer, fabricated by HL or NIL, deposited over inorganic substrate; active film on top of it; $\Lambda$ : Grating period.

Most of the solution processable materials thus far investigated ${ }^{1,2}$ for TFOL fabrication purposes belong to one of the two following classes: (i) organic semiconductors - mainly polymers, but also oligomers, dendrimers and small molecules, all of which were used as neat films; and (ii) conventional aromatic dyes dispersed typically at 3-5 wt $\%$ in an inert solid matrix among which thermoplastic polymers, such as polystyrene (PS) or poly methylmethacrylate (PMMA), are the most convenient for processing. Materials from class (i) have shown the lowest thresholds (often below 1 $\left.\mathrm{kW} / \mathrm{cm}^{2}\right),{ }^{7-13}$ in some cases low enough to enable pumping with compact sources or even with LEDs, ${ }^{12,13}$ but their photostabilities in air are generally poor, so necessitate protection of the device from molecular oxygen. On the other hand, TFOLs based on certain materials from class (ii), particularly those including highly photostable dyes of the pyrromethene $(\mathrm{PM})^{14}$ or the perylenediimide (PDI) ${ }^{15-17}$ families dispersed in PS or PMMA, have shown operational lifetimes as long as $10^{5}$ pump pulses, ${ }^{15}$ whereas their thresholds are often high, typically $10-100 \mathrm{~kW} / \mathrm{cm}^{2} 1,2,14,15$ and at a lowest value of $3 \mathrm{~kW} / \mathrm{cm}^{2}$ in a PDI compound. ${ }^{16}$ The higher thresholds of dye-doped materials, with respect to organic semiconductors, is because of high dilution in the matrix, which on the other hand is necessary to prevent aggregative excited state annihilation. An attractive property of PDI-doped PS films is that their thermal and optical properties keep unaltered after being heated up to temperatures of around $155^{\circ} \mathrm{C}$. This has allowed fabricating DFB lasers with the gratings engraved directly on the active materials, ${ }^{17}$ by using thermal-NIL (geometry (b) according to Figure 1), which have shown high photostability and thresholds as low as $8 \mathrm{~kW} / \mathrm{cm}^{2}$, which is a rather low value for an imprinted laser. Note that thermal-NIL cannot be used to imprint most of the typical materials used for TFOLs since their optical properties degrade under high temperatures. ${ }^{1,2}$ A limitation of PDIs is that up to recently, only derivatives without substituents at the bay positions (bay-unsubstituted PDIs, $u$-PDIs), have shown sufficiently good performance to produce TFOLs with high photostability and reasonable threshold. ${ }^{15-17}$ These compounds show ASE at around the same wavelength $(579 \mathrm{~nm})$, independently on the type of substituent attached to the $N$ atoms, and it cannot be red-shifted by increasing the dye concentration in the matrix, as can be done with other materials, since the range of PDI concentrations which provides ASE is very limited. ${ }^{16}$ Therefore, designing PDIs emitting at longer wavelengths and showing good laser characteristics is of great interest. In previous studies of bay-substituted PDIs, $b$-PDIs, PL quantum yield and ASE performance was rather poor, due to the substituent induced PDI core distortion. ${ }^{18}$

In this paper we describe last advances towards the development of novel active laser materials showing high performance simultaneously at the various mentioned parameters. Particularly, we report ASE and DFB results for a $b$ PDI derivative, denoted as $b$-PDI-1, and compare them to those compared of two $u$-PDI derivatives ( $u$-PDI-C6 and $u$ - 
PDI-O) used previously in the context of organic lasers (see chemical structures in Figure 2). Results for a novel family of carbon-bridged oligo- $p$-phenylenevinylenes (COPVn, with $n=1-6$, chemical structures in Figure 3) are also presented.

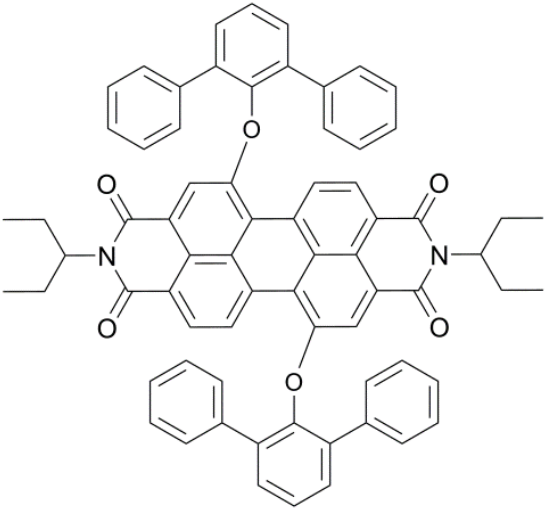

(a)

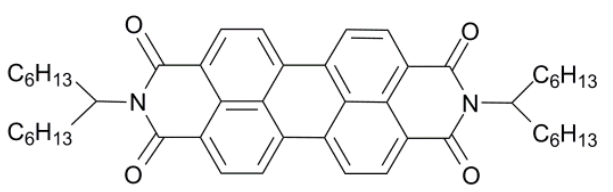

(b)

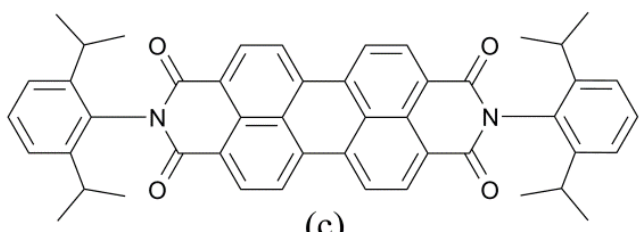

(c)

Figure 2. Chemical structures of various perylenedimide (PDI) dyes: (a) bay substituted $b$-PDI-1; (b) bay unsubstituted $u$-PDI-C6; and (c) bay unsubstituted $u$-PDI-O.

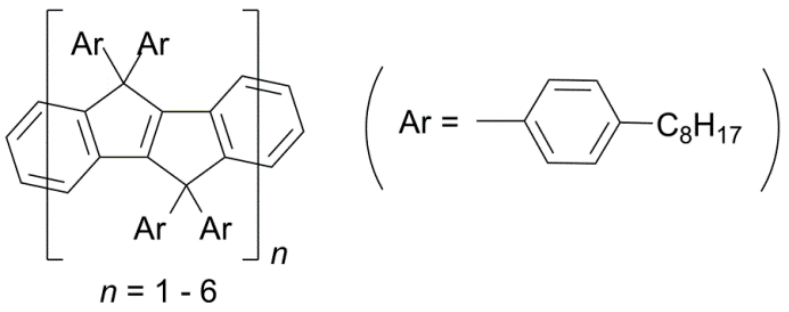

Figure 3. Chemical structure of carbon-bridged oligo( $p$-phenylenevinylene)s, COPV $n$, with $n=1$ to 6 .

\section{EXPERIMENTAL SECTION}

The synthetic procedures to prepare $b$-PDI- $1^{19}$ and COPVs ${ }^{20}$ have been previously reported. The rest of the compounds are commercially available and used as received. Thin films of PS doped with a laser dye were prepared by spin-coating over substrates. The percentage of PS in the solvent (toluene) was adjusted to control the film thickness to between 300 and $1100 \mathrm{~nm}$, depending on the material. Commercially available fused silica (FS) substrates were used for absorption, PL and ASE measurements. DFB lasers with geometries (a) and (c), according to Figure 1, were fabricated by depositing the active film on a substrate of a different nature as required, with a surface relief grating previously recorded using HL or thermal-NIL. Grating dimensions were $2.5 \mathrm{~cm} \times 2.5 \mathrm{~cm}$ and $2 \mathrm{~mm} \times 2 \mathrm{~mm}$ for HL and thermal-NIL gratings, respectively. Resonators for geometry (c) devices, based on either dichromated gelatine (DCG) or dichromated poly(vinyl alcohol) (DCPVA) photoresist layers deposited over FS, were fabricated as previously described. ${ }^{21,22}$ Resonators for geometry (a) devices were prepared by thermal-NIL and subsequent etching on either $\mathrm{FS}$ or $\mathrm{SiO}_{2}$ layers grown by thermal oxidation of silicon wafers, ${ }^{23}$ or by $\mathrm{HL}$ and etching on glass. ${ }^{24}$

ASE and DFB characterizations were performed under optical excitation with a pulsed Nd:YAG (YAG: yttrium aluminum garnet) laser (10 ns pulse width, $10 \mathrm{~Hz}$ repetition rate). For ASE, the pump beam (a stripe of dimensions 3.5 $\mathrm{mm} \times 0.5 \mathrm{~mm}$ ) was incident perpendicularly over the sample surface, and the emitted light was collected from the film edge with a fiber spectrometer (resolution $1.3 \mathrm{~nm}$ ). For DFB characterization, the pump beam over the sample (elliptical with a minor axis of $1.1 \mathrm{~mm}$ ) was incident at $\sim 20^{\circ}$ with respect to the normal to the film plane. The emitted light was 
collected by means of a $0.13 \mathrm{~nm}$ resolution fiber spectrometer, in a direction perpendicular to the sample surface. The pump size value was chosen as to be sufficiently large to ensure that the obtained laser threshold parameter expressed in power or energy density units is a useful parameter for the sake of comparison. ${ }^{25}$ For both ASE and DFB studies, $\lambda_{\text {pump }}$ was $355 \mathrm{~nm}$ for COPV1 and COPV2, $532 \mathrm{~nm}$ for COPV5, COPV6 and for all PDIs, and $436 \mathrm{~nm}$ (provided by a Raman cell pumped with the $532 \mathrm{~nm}$ line of the Nd:YAG laser) for COPV3 and COPV4.

\section{RESULTS AND DISCUSSION}

\subsection{Bay-substituted perylene.}

$b$-PDI-1 is a bay-substituted PDI presenting two sterically hindering diphenylphenoxy groups at the 1,7 position and ethylpropyl substituents in the imide positions, see Figure 2(a). Its PL spectrum, when dispersed in PS, has a shape similar to that obtained in liquid solution, up to $b$-PDI-1 contents of around $37 \times 10^{-5}$ mol of PDI per gram of PS (equivalent to $27 \mathrm{wt} \%$ ) and its intensity increases almost linearly, indicating that no significant PL quenching occurs. ${ }^{19}$ This property is a significant feature in comparison to other PDIs, whose PL starts quenching at concentrations of 3-5 $\mathrm{wt} \%$, effect generally attributed to the formation of aggregated species. $b$-PDI-1 PL quantum yield (PLQY) in liquid solution is close to 1 , which is signature of an undistorted core, in contrast to most bay substituted PDIs reported in the literature whose PLQY are significantly lower due to the core distortion induced by the bay substituents. ${ }^{18}$

ASE spectra for PS films with different $b$-PDI-1 contents are shown in Figure 4(a). The ASE spectrum red-shifts upon the concentration increase. An important consequence of the capability of introducing large amounts of PDI in the films without sever PL quenching and therefore showing ASE, is that the ASE wavelength can be tuned over a relatively wide range (around $20 \mathrm{~nm}$, from $\lambda_{\mathrm{ASE}}=610$ to $\lambda_{\mathrm{ASE}}=630 \mathrm{~nm}$ ). This is a clear advantage over $u$-PDIs (see data for $u$-PDI-O in Figure 4(a)) for which ASE emission appears at around $\lambda_{\mathrm{ASE}}=579 \mathrm{~nm}$ and it can only be slightly shifted by a few $\mathrm{nm}$. This is because the PDI concentration cannot be increased further than $3 \mathrm{wt} \%$, otherwise the film PL would quench and the ASE performance would degrade.

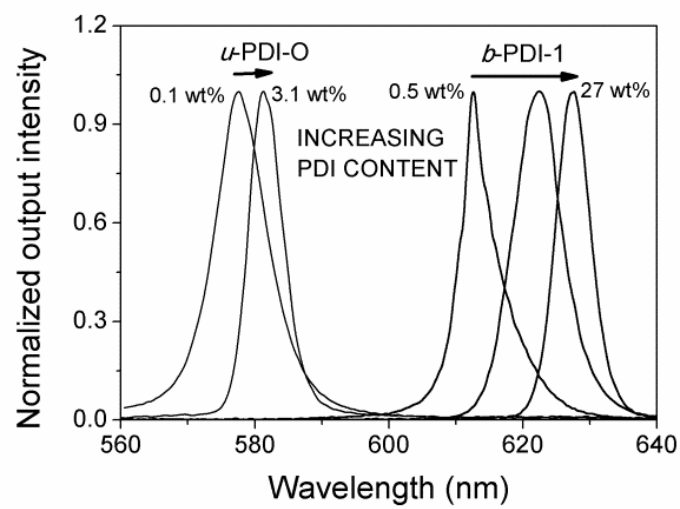

(a)

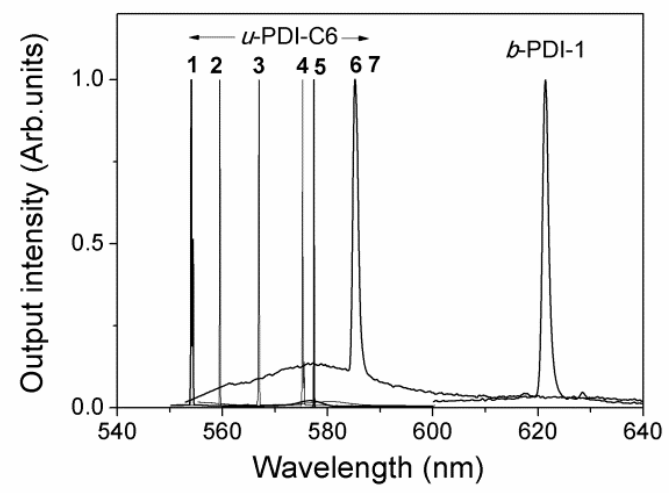

(b)

Figure 4. (a) ASE spectra for PS films containing either $u$-PDI-O or $b$-PDI-1 at various doping ratios, illustrating the ASE spectrum red-shift upon dye concentration increase. (b) DFB spectra of devices based on PS doped with either $u$-PDI-C6 $(0.5 \mathrm{wt} \%)$ or with $b$-PDI-1 (5 wt\%). Lasers based on $u$-PDI-C6 labeled 1-5 have $\mathrm{SiO}_{2}$ resonators and geometry (a), according to Figure 1, with parameters $\Lambda=368 \mathrm{~nm}, d=65 \mathrm{~nm}$ and $h$ values of 300, 330, 430 and $780 \mathrm{~nm}$, respectively; Lasers based on $u$-PDI-C6 labeled 6-7 have photoresist resonators and geometry (c), with different device parameters (see text for details).

With regards to the $b$-PDI-1 ASE threshold, it is observed that it decreases with increasing $b$-PDI- 1 concentration to a minimum of $7 \mathrm{~kW} / \mathrm{cm}^{2}$, at PDI contents between $6 \times 10^{-5}$ and $13 \times 10^{-5}$ mol of $b$-PDI-1 per gram of PS (equivalent to around $8 \mathrm{wt} \%) .{ }^{19}$ The PLQY for these films is around $60 \%$. This ASE threshold is comparable to those of $u$-PDI-C6 and $u$-PDI-O, which have shown values of $8-10^{15,17}$ and $3 \mathrm{~kW} / \mathrm{cm}^{2}{ }^{16}$ respectively. The photostability of $b$-PDI-1 in air is high when the doping rate is small. For example, the photostability half-life, $\tau_{1 / 2-\mathrm{ASE}}$, defined as the time (or the number of 
pump pulses) at which the ASE intensity decays to half of its initial value, under a pump intensity two times above threshold, is $\tau_{1 / 2-\mathrm{ASE}}=5 \times 10^{4}$ pump pulses, for the film doped with $0.5 \mathrm{wt} \%$. This value is much superior to that of previously investigated $b$-PDIs, whose ASE lasted just for a few minutes ${ }^{18}$ and only slightly inferior to that obtained with films containing $u$-PDI-O and $u$-PDI-C6 at similar doping rates, excited under similar conditions $\left(\tau_{1 / 2-\mathrm{ASE}} \sim 1 \times 10^{5}\right.$ pump pulses). ${ }^{15,16}$ It was found that the $b$-PDI-1 photostability decreases with increasing concentration $\left(\right.$ at $8 \mathrm{wt} \%, \tau_{1 / 2-\mathrm{ASE}}=$ $1.5 \times 10^{4}$ pump pulses). This establishes a trade-off between photostability and threshold which should be taken into account when choosing the optimal concentration for a given application.

A DFB laser based on a $b$-PDI-1-doped PS films and a resonator fabricated by HL on DCG with a geometry such as the one in Figure 1(c), was prepared. The DFB spectrum is shown in Figure 4(b), which includes also the spectra of lasers based on $u$-PDI-C6 DFBs fabricated by the same method and with similar geometry, and also some others prepared over fused silica with thermal-NIL and subsequent etching, with geometry (a) according to Figure 1. Wavelength tunability on the later devices (labelled as 1-5) was obtained by changing film thickness. ${ }^{23}$ The ones based on photoresist resonators (devices 6-7) had different device parameters: $\Lambda=378 \mathrm{~nm}, d=80 \mathrm{~nm}, h=1100 \mathrm{~nm}$, DCG as resonator material, and operation in the first order transverse electric mode $\mathrm{TE}_{1}$ (for device 6 ) ${ }^{21}$ and $\Lambda=370 \mathrm{~nm}, d=80 \mathrm{~nm}, h=$ $600 \mathrm{~nm}$, dichromated polyvinyl alcohol (DCPVA), as resonator material, and operation in the fundamental transverse electric mode TE0 (for device 7). ${ }^{22}$ DFBs fabricated by NIL have shown lower thresholds. For example, for device 5, whose emission is close to $\lambda_{\mathrm{ASE}}$, at which the gain is maximum, it is around $12 \mathrm{~kW} / \mathrm{cm}^{2}$. This is in contrast to the 55 $\mathrm{kW} / \mathrm{cm}^{2}$ value found for the device based on $b$-PDI-1, which also emits close to its corresponding ASE wavelength, despite its ASE threshold is similar to that of $u$-PDI-C6.

\subsection{Carbon-bridged oligo-p-phenylenevinylenes.}

Very recently, a novel family of PV oligomers with carbon bridges linking their rings (COPVn, with $n=1-6$, Figure 3), has shown an exceptional performance simultaneously on the various important parameters for laser applications. ${ }^{26}$ The COPV $n$ molecules dispersed in PS films show ASE from $385 \mathrm{~nm}$ to $585 \mathrm{~nm}$ (see Figure 5(a)), thus covering the whole visible spectrum. COPV $n$-based DFB lasers with geometries (a) and (c), according to Figure 1, have been fabricated with all these compounds, using geometries (a) and (c) according to Figure 1 (illustrative spectra are shown in Figure 5b).

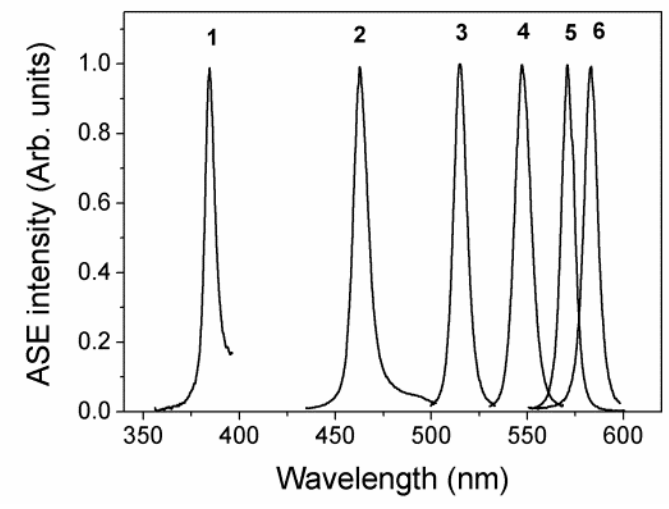

(a)

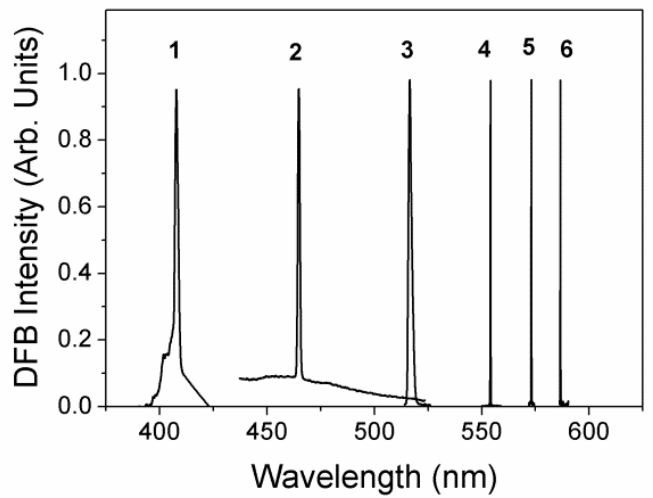

(b)

Figure 5. (a) ASE spectra for PS films containing $2 \mathrm{wt} \%$ of COPV $n(n=1-6)$. The label number in each spectrum refers to $n$; (b) DFB spectra of devices based on COPVn-doped PS. Lasers based on COPV5-6 have $\mathrm{SiO}_{2}$ resonators and geometry (a), according to Figure 1, with parameters $\Lambda=368 \mathrm{~nm}, d=95 \mathrm{~nm}$ and $h=630 \mathrm{~nm}$ (devices 5) and $\Lambda=380 \mathrm{~nm}, d=75 \mathrm{~nm}$ and $h=490 \mathrm{~nm}$ (devices 6); Lasers based on COPV2-4 have photoresist resonators and geometry (c), with parameters: $\Lambda=$ $296 \mathrm{~nm}, d=40 \mathrm{~nm}$ and $h=380 \mathrm{~nm}$ (device 2); $\Lambda=320 \mathrm{~nm}, d=80 \mathrm{~nm}$ and $h=650 \mathrm{~nm}$ (device 3 ); and $\Lambda=350 \mathrm{~nm}, d=60$ $\mathrm{nm}$ and $h=640 \mathrm{~nm}$ (device 4); COPV1-based DFB laser has a glass resonator and geometry (a), according to Figure 1, and parameters $\Lambda=270 \mathrm{~nm}, d=70 \mathrm{~nm}$ and $h=610 \mathrm{~nm}$. 
Remarkably, devices based on COPV3-6 have shown very low thresholds to a minimum of $0.7 \mathrm{~kW} / \mathrm{cm}^{2}$ in some cases, being this value close to the requirements to pump with LEDs. Another prominent feature is the exceptional device operational lifetime ( $>10^{5}$ pump pulses, for $n=3-6$ ), as a consequence of the outstanding photostability of these compounds. Also noticeable is their high thermal stability (decomposition temperatures are above $400{ }^{\circ} \mathrm{C}$ ), so it is expected that they will allow thermal-NIL imprinting of the DFB resonators directly onto the active film (geometry (b), according to Figure 1), as demonstrated for PDI-doped PS. ${ }^{17}$

\section{CONCLUSIONS}

ASE and DFB laser applications of high performing materials based on novel laser dyes of two different classes, perylenediimides and oligo- $p$-phenylenevinylenes (OPVs), both dispersed in films of PS, used as passive matrix, have been reported. Films containing a new bay-substituted PDI compound ( $b$-PDI-1) show efficient ASE emission at wavelengths between 610 and $630 \mathrm{~nm}$, widening the use of PDIs in laser applications, up to now restricted to bayunsubstituted PDIs, all with ASE at around $579 \mathrm{~nm}$. Results on a new class of compounds, carbon-bridged OPVs, denoted as COPV $n$ (with $n=1$ to 6) have been described. Films of COPV $n$-doped PS undergo ASE from $385 \mathrm{~nm}$ to 583 $\mathrm{nm}$, depending on the oligomer length, with remarkably low threshold and high photostability. Second-order DFB lasers with standard gratings (single period), have shown single mode emission, wavelength tunability across the visible spectrum, long operational lifetimes of $>10^{5}$ pump pulses, outstandingly larger than previously reported phenylenevinylene oligomers or polymers, and thresholds close to pumping requirements with light-emitting diodes, i.e. only $0.7 \mathrm{~kW} / \mathrm{cm}^{2}$, which is as far as we know the lowest values reported to date for dye-doped materials. It is also remarkable that the two types of systems discussed show high thermal stability, thus allowing the use of thermal nanoimprint lithography to engrave the DFB resonators directly onto the active films. This constitutes an important aspect for the prospect of preparing mechanically flexible inexpensive devices.

\section{ACKNOWLEDGEMENTS}

This work was supported by the Spanish Government (MINECO) and the European Community (FEDER) through grant no. MAT-2011-28167-C02. M. Morales-Vidal has been partly supported by a MINECO FPI fellowship (no. BES-2009020747). Prof. E. Nakamura and Dr. H. Tsuji at the Univeristy of Tokyo (Japan) are acknowledged for synthesizing and supplying the COPVn compounds. Prof. A. Sastre-Santos and Prof. F. Fernández-Lázaro at Miguel Hernández University (Spain) are acknowledged for synthesizing and supplying the $b$-PDI-1 derivative.

\section{REFERENCES}

[1] Chénais, S. and Forget, S., "Recent advances in solid-state organic lasers," Polym. Int. 61, 390-406 (2012).

[2] Grivas, C. and Pollnau, M., "Organic solid-state integrated amplifiers and lasers," Laser Photonics Rev. 6, 419-462 (2012).

[3] Liu, X., Stefanou, P., Wang, B., Woggon, T., Mappes, T. and Lemmer, U., "Organic semiconductor DFB laser as excitation source in Raman spectroscopy," Opt. Express 21, 28941-28947 (2013).

[4] Vannahme, C., Klinkhammer, S., Lemmer, U. and Mappes, T., "Plastic lab-on-a-chip for fluorescence excitation with integrated organic semiconductor lasers," Opt. Express 19, 8179-8186 (2011).

[5] Wang, Y., Morawska, P. O., Kanibolotsky, A. L., Skabara, P. J., Turnbull, G. A. and Samuel, I. D. W., "LED pumped polymer laser sensor for explosives," Laser Photonics Rev. 7, L71-L76 (2013).

[6] Heydari, E., Buller, J., Wischerhoff, E., Laschewsky, A., Döring, S. and Stumpe, J., "Label-free biosensor based on an all-polymer DFB laser," Adv. Optical Mater. 2, 137-141 (2014).

[7] Karnutsch, C., Gýrtner, C., Haug, V., Lemmer, U., Farrell, T., Nehls, B. S., Scherf, U., Wang, J., Weimann, T., Heliotis, G., Pflumm, C., deMello J. C. and Bradley, D. D. C., "Low threshold blue conjugated polymer lasers with first- and second-order distributed feedback," Appl. Phys. Lett. 89, 201108 (2006). 
[8] Karnutsch, C., Pflumm, C., Heliotis, G., deMello, J. C., Bradley, D. D. C., Wang, J., Weimann, T., Haug, V., Gärtner, C. and Lemmer, U., "Improved organic semiconductor lasers based on a mixed-order distributed feedback resonator design," Appl. Phys. Lett. 90, 131104 (2007).

[9] Yap, B. K., Xia, R., Campoy-Quiles, M., Stavrinou, P. N. and Bradley, D. D. C., "Simultaneous optimization of charge-carrier mobility and optical gain in semiconducting polymer films," Nature Mater. 7, 376-380 (2008).

[10] Xia, R., Lai, W-L., Levermore, P. A., Huang, W. and Bradley, D.D. C., "Low-threshold distributed-feedback lasers based on pyrene-cored starburst molecules with 1,3,6,8-attached oligo(9,9-dialkylfluorene) arms," Adv. Funct. Mater. 19, 2844-2850 (2009).

[11] Yang, Y., Turnbull, G. A. and Samuel, I. D., "Hybrid optoelectronics: a polymer laser pumped by a nitride lightemitting diode," Appl. Phys. Lett. 92, 163306 (2008).

[12] Herrnsdorf, J., Wang, Y., McKendry, J. J. D., Gong, Z., Massoubre, D., Guilhabert, B., Tsiminis, G., Turnbull, G. A., Samuel, I. D. W., Laurand, N., Gu, E. and M. D., Dawson, "Micro-LED pumped polymer laser: a discussion of future pump sources for organic lasers," Laser Photonics Rev. 7, 1065-1078 (2013).

[13] Tsiminis, G., Wang, Y., Kanibolotsky, A. L., Inigo, A. R., Peter, Skabara, J., Samuel, I. D. W. and Turnbull, G. A., "Nanoimprinted organic semiconductor laser pumped by a light-emitting diode," Adv. Mater. 25, 2826-2830 (2013).

[14] Yang, Y., Goto, R., Omi, S., Yamashita, K., Watanabe, H., Miyazaki, M. and Oki, Y., "Highly photostable dye doped solid-state distributed-feedback (DFB) channeled waveguide lasers by a pen-drawing technique," Opt. Express 18, 22080-22089 (2010).

[15] Navarro-Fuster, V., Calzado, E. M., Boj, P. G., Quintana, J. A., Villalvilla, J. M., Díaz-García, M. A., Trabadelo, V., Juarros, A., Retolaza, A. and Merino, S, "Highly photostable organic distributed feedback laser emitting at 573 nm," Appl. Phys. Lett. 97, 171104 (2010).

[16] Ramírez, M. G., Morales-Vidal, M., Navarro-Fuster, V., Boj, P. G., Quintana, J. A., Villalvilla, J. M., Retolaza, A., Merino, S. and Díaz-García, M. A., "Improved performance of perylenediimide-based lasers," J. Mater. Chem. C. 1, 1182-1191 (2013).

[17] Ramírez, M. G., Boj, P. G., Navarro-Fuster, V., Vragovic, I., Villalvilla, J. M., Alonso, I., Trabadelo, V., Merino, S. and Díaz-García, M. A., "Efficient organic distributed feedback lasers with imprinted active films," Opt. Express 19, 22443-22454 (2011).

[18] Calzado, E.M., Villalvilla, J.M., Boj, P.G., Quintana, J.A., Gómez, R.., Segura, J.L. and Díaz-García, M.A., "Effect of structural modifications in the spectral and laser properties of perylenediimide derivatives," J. Phys. Chem. C $111,13595-13605$ (2007).

[19] Ramírez, M. G., Pla, S., Boj, P. G., Villalvilla, J. M., Quintana, J. A., Díaz-García, M. A,. Fernández-Lázaro, F. and Sastre-Santos, A, "1,7-Bay-substituted perylenediimide derivative with outstanding laser performance," Adv. Optical Mater. 1, 933-938 (2013).

[20]Zhu, X., Tsuji, H., López-Navarrete, J. T., Casado, J. and Nakamura, E., "Carbon-bridged oligo(phenylenevinylene)s: stable $\pi$-systems with high responsiveness to doping and excitation," J. Am. Chem. Soc. 134, 19254-19259 (2012).

[21] Ramírez, M. G., Quintana, J. A., Villalvilla, J. M., Boj, P. G., Retolaza, A., Merino, S. and Díaz-García, M. A., "Perylenediimide-based distributed feedback lasers with holographic relief gratings on dichromated gelatine," J. Appl. Phys. 114, 033107 (2013).

[22] Ramírez, M. G., Villalvilla, J. M., Quintana, J. A., Boj, P. G. and Díaz-García, M. A., "Distributed feedback lasers based on dichromated poly(vinyl alcohol) reusable surface-relief gratings," Opt. Mater. Express 4, 733-738 (2014).

[23] Navarro-Fuster, V., Vragovic, I., Calzado, E. M., Boj, P. G., Quintana, J. A., Villalvilla, J. M., Retolaza, A., Juarros, A., Otaduy, D., Merino, S. and Díaz-García, M, A., "Film thickness and grating depth variation in organic second-order distributed feedback lasers," J. Appl. Phys. 112, 043104 (2012).

[24] Calzado, E. M. Villalvilla, J. M., Boj, P. G., Quintana, J. A., Postigo, P. A. and Díaz-García, M. A., "Blue surfaceemitting distributed feedback lasers based on TPD-doped films, " Appl. Opt. 49, 463-470 (2010).

[25] Calzado, E. M., Villalvilla, J. M., Boj, P. G., Quintana, J. A., Navarro-Fuster, V., Retolaza, A., Merino, S. and Díaz-García, M. A., "Influence of the excitation area on the thresholds of organic second-order distributed feedback lasers," Appl. Phys. Lett. 101, 223303 (2012).

[26] Morales-Vidal, M., Boj, P.G., Villalvilla, J.M., Quintana, J.A., Yan, Q. Lin, N., Zhu, X., Ruangsupapichat, N., Casado, J., Tsuji, H. Nakamura, H. and Díaz-García, M.A., "Carbon-bridged oligo(p-phenylenevinylene) for photostable and broadly tunable solution processable thin film organic lasers," Nature Commun. (accepted). 